\title{
Graph Matching Iris Image Blocks with Local Binary Pattern
}

\author{
Zhenan Sun, Tieniu Tan, and Xianchao Qiu \\ Center for Biometrics and Security Research, \\ National Laboratory of Pattern Recognition, Institute of Automation, \\ Chinese Academy of Sciences, P.O. Box 2728, Beijing, 100080, P.R. China \\ \{znsun, tnt, xcqiu\}@nlpr.ia.ac.cn
}

\begin{abstract}
Iris-based personal identification has attracted much attention in recent years. Almost all the state-of-the-art iris recognition algorithms are based on statistical classifier and local image features, which are noise sensitive and hardly to deliver perfect recognition performance. In this paper, we propose a novel iris recognition method, using the histogram of local binary pattern for global iris texture representation and graph matching for structural classification. The objective of our idea is to complement the state-of-the-art methods with orthogonal features and classifier. In the texture-rich iris image database UPOL, our method achieves higher discriminability than state-of-the-art approaches. But our algorithm does not perform well in the CASIA database whose images are less textured. Then the value of our work is demonstrated by providing complementary information to the state-of-the-art iris recognition systems. After simple fusion with our method, the equal error rate of Daugman's algorithm could be halved.
\end{abstract}

\section{Introduction}

Iris-based identity authentication has many important applications in our networked society. Since the last decade, much research effort has been directed towards automatic iris recognition. Because the distinctive information of iris pattern is preserved in the randomly distributed micro-textures, constituted by freckles, coronas, stripes, furrows, etc., most of the state-of-the-art iris recognition algorithms are based on the local features of iris image data. Typical iris recognition methods are Gabor-based phase demodulation [1], local intensity variations [2] and wavelet zero-crossing features [3], etc. However, the minutiae-based iris representation is sensitive to noise, such as the occlusions of eyelids and eyelashes, non-linear deformations, imperfect localization or alignment, etc. So it is a straightforward idea to complement local features based methods with global structural features.

In our early attempt [4], blobs of interest are segmented from the iris images for spatial correspondence. Experimental results demonstrated the effectiveness of combining local statistical features and global structural features. But the segmentation of foreground regions in some poor quality images, e.g. defocused iris images, is a difficult problem. In addition, both the feature extraction and matching of blob patterns [4] were not very efficient. 
We think the distinctiveness of an iris pattern relies on the statistical features of local image regions and the spatial relationship between these regions. Motivated by the fact that the literature has ignored the global topological information in iris data, the iris features are represented from both local and global aspects in this paper: local binary pattern (LBP operator) is adopted to characterize the iris texture in each image block, and all localized image blocks are used to construct a global graph map. Then the similarity between two iris images is measured by a simple graph matching scheme.

The novelty of this paper is that both LBP and image blocks based graph matching are introduced for the first time to iris recognition and in a fusion manner. Another contribution is that our method is good complement of the state-of-the-art iris recognition systems with orthogonal features and classifiers.

The remainder of this paper is organized as follows. Section 2 introduces the LBPbased attribute graph representation scheme. The graph matching method, aiming to find the correspondence between two iris images, is provided in Section 3. Experimental results on two publicly available iris databases are reported in Section 4. Section 5 concludes this paper.

\section{LBP-Based Iris Feature Representation}

LBP describes the qualitative intensity relationship between a pixel and its neighborhoods, which is robust, discriminant, and computationally efficient so it is well suited to texture analysis [5]. We choose LBP to represent iris image blocks'

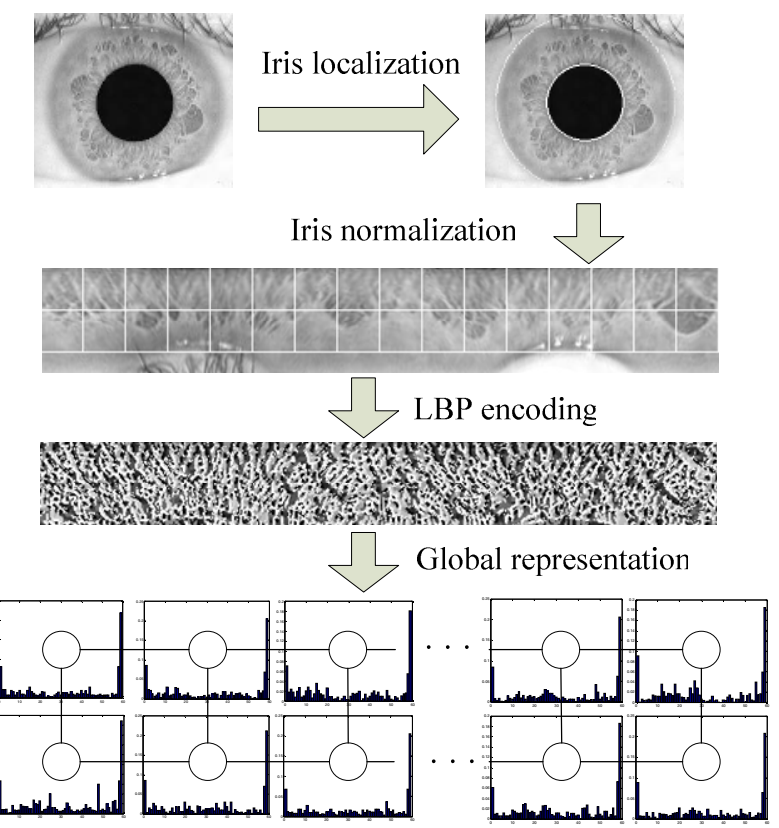

Fig. 1. The flowchart of the LBP-based iris graph representation 
distinctive information because iris pattern could be seem as texture constituted by many minute image structures. This is the first attempt in literature to use LBP for iris recognition.

The whole procedure of iris feature extraction is illustrated in Figure 1. Firstly, the input iris image should be preprocessed and normalized to correct the position and scale variations before iris feature extraction and matching. In our paper, the resolution of the normalized iris image is 80 by 512 . To exclude the possible occlusions of eyelids and eyelashes, we divide the upper region of the normalized iris image into $2 * 16=32$ blocks, and each block has the size 32 by 32 . For each block in the normalized iris image, an eight-neighborhood uniform LBP histogram with radius 2 (59 bins) [5] may be obtained. In our labeled graph representation of iris pattern, each manually divided image block is regarded as a graph node, associated with the attributes of the local region's LBP histogram. And the spatial layout of these image blocks is used to model the structural relations among the nodes. Finally, a graph with 32 nodes is constructed as the template of each iris image (Figure 1).

\section{Graph Matching Iris Features}

Because an iris pattern has randomly distributed minute features, varying from region to region, the basic idea underlying our graph matching scheme is qualitative corresponding theory. For each block of an iris image, it should be the most similar to the corresponding block in another image if these two iris images $(A$ and $B$ ) are from the same eye. So we only need to count the number of the best matching block pairs, which are required to satisfy two conditions:

1) The matching blocks have the minimal distance based on a similarity metric, i.e. $\min _{j} \operatorname{Distance}\left(A^{i}, B^{j}\right) \forall i, j=1,2, \cdots, 32$. In addition, their distance should be lower than a given threshold $C_{T h}$.

2) The matching blocks have the same topological layout, i.e. the corresponding blocks have the same spatial position in the graph representation.

Compared with parametric classification principles, non-parametric classification strategy is more flexible and avoids the assumption on the distribution of input data. In this paper, the Chi-square statistic is used to evaluate the dissimilarity between two LBP histograms $H A^{i}\left\{H A_{1}^{i}, H A_{2}^{i}, \cdots, H A_{59}^{i}\right\}$ and $H B^{j}\left\{H B_{1}^{j}, H B_{2}^{j}, \cdots, H B_{59}^{j}\right\}$ :

$$
\chi^{2}\left(H A^{i}, H B^{j}\right)=\sum_{k=1}^{59} \frac{\left(H A_{k}^{i}-H B_{k}^{j}\right)^{2}}{H A_{k}^{i}+H B_{k}^{j}}
$$

Because it is possible that $H A_{k}^{i}+H B_{k}^{j}=0$, the summation only includes the nonzero bins. Suppose the LBP features of the two iris images are $H A\left\{H A^{1}, H A^{2}, \cdots, H A^{32}\right\}$ and $H B\left\{H B^{1}, H B^{2}, \cdots, H B^{32}\right\}$ respectively, so their matching score $S$ is computed as follows: 


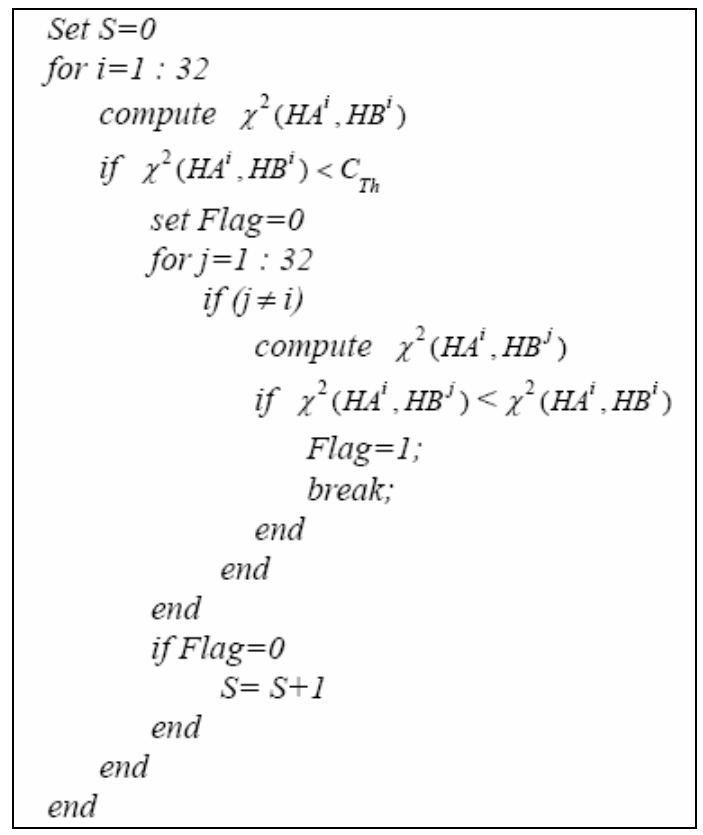

Fig. 2. The pseudo code of the graph matching of LBP features

$C_{T h}$ is a constant value learned from the training set. For genuine corresponding block pairs, the probability of their Chi Square lower than the $C_{T h}$ should be more than 0.8 . The matching score $S$ has the range from 0 to 32 , and could be normalized as $S / 32$ to obtain a uniform output for fusion. The higher the matching score, the higher the probability of the two images being from the same eye.

\section{Experiments}

To evaluate the effectiveness of our method for iris recognition, two publicly available iris databases, UPOL [6] and CASIA [7] are used as the test datasets. The first one is constituted by European volunteers, captured under visible lighting. And the second one mainly comes from Chinese volunteers, captured under infrared illumination.

The UPOL iris database [6] includes 384 iris images from 64 persons. All possible intra-class and inter-class comparisons are made to estimate the genuine distribution and imposter distribution respectively, i.e. totally 384 genuine samples and 73,152 imposter samples. The distribution of these matching results is shown in Figure 3. For the purpose of comparison, two state-of-the-art iris recognition algorithms, Daugman's [1] and Tan's [2], are also implemented on the same dataset. Although these three methods all achieve perfect results, i.e. without false accept and false reject, our 
method obtains higher discriminating index $\left(_{\mathrm{DI}}=\frac{\left|m_{1}-m_{2}\right|}{\sqrt{\left(\delta_{1}^{2}+\delta_{2}^{2}\right) / 2}}\right.$, where $m_{1}$ and $\delta_{1}^{2}$ denote the mean and variance of intra-class Hamming distances, and $m_{2}$ and $\delta_{2}^{2}$ denote the mean and variance of inter-class Hamming distances.) [1] (See Fig. 3).

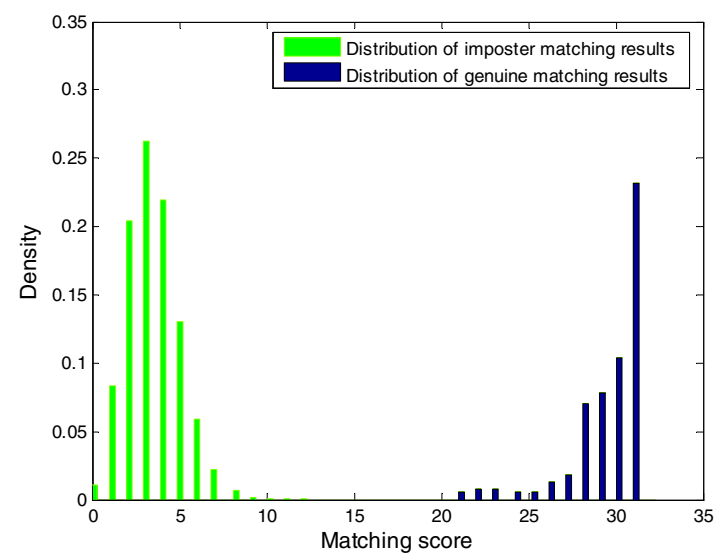

Fig. 3. The distribution of matching results of our method on the UPOL database. The DI is 15.2. In contrast, the DI of Daugman's method [1] is 7.9 and that of Tan's [2] is 8.6.

The CASIA database is the largest open iris database [7] and we only use the subset described in [2] for performance evaluation. There are totally 3,711 intra-class comparisons and 1,131,855 inter-class comparisons. The distribution of the matching results of our method is shown in Fig. 4. The maximal inter-class matching score is 12. We can see that the comparison results of genuine and imposter are well separated by our method although they overlap each other in a minor part. The ROCs (receiver operating curve) of the three methods are shown in Fig. 5. It is clear that our method does not perform as well as the state-of-the-art methods on this dataset. We think the main reason is that the texture information of Asian subjects is much less than that of the Europeans, especially on the regions far from the pupil, but the effectiveness of LBP histogram heavily depends on the abundant micro-textures. The main purpose of this paper is to develop the complementary global features, along with the commonlyused local features, to improve the accuracy and robustness of an iris recognition system. The score-level fusion results based on Sum rule are shown in Fig. 5 and Table 1. After introducing the matching results of LBP features and structural classifier, the equal error rate (EER) of Daugman's method [1] is halved. Similarly, about $30 \%$ EER is reduced from Tan's method [2] (Table 1). Comparatively, combining two local features based methods does not show significant improvement (Table 1). The disadvantage of our method is that the graph matching diagram is time consuming because of many iterations, but it still could be implemented in real time. In addition, if we adopt a cascading scheme like that described in [4], the computational complexity could be considerably reduced. 


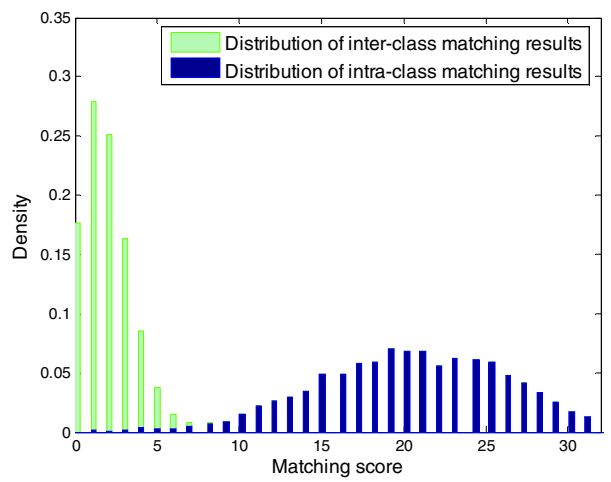

Fig. 4. The distribution of matching results of our method on CASIA database

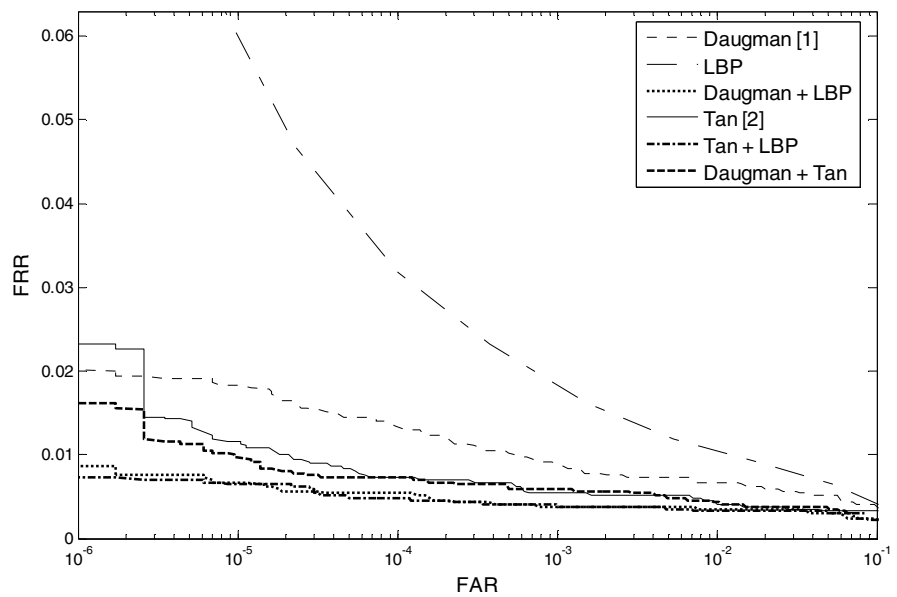

Fig. 5. Comparison of ROC curves of different iris recognition methods on CASIA database

Table 1. Comparison of recognition accuracy of various recognition schemes

\begin{tabular}{|c|c|c|}
\hline Recognition scheme & DI & EER \\
\hline Daugman [1] & 4.74 & $0.70 \%$ \\
\hline Tan [2] & 5.36 & $0.51 \%$ \\
\hline LBP & 4.46 & $0.86 \%$ \\
\hline Daugman + LBP & 5.31 & $0.37 \%$ \\
\hline Tan + LBP & 5.51 & $0.32 \%$ \\
\hline Daugman + Tan & 5.23 & $0.49 \%$ \\
\hline
\end{tabular}




\section{Conclusions}

In this paper, a new iris recognition method has been proposed to complement the state-of-the-art approaches. LBP operator, which is successfully applied to texture analysis and face recognition, is firstly employed to represent the robust texture features of iris images. A novel graph matching scheme is exploited to measure the similarity between two iris images. Experimental results on two publicly available iris image databases, UPOL and CASIA, illustrated the effectiveness of our method. The largest advantage of our method is its robustness against noise or occlusions in iris images because our algorithm only needs to match only a fraction of all image blocks to authenticate a genuine. Comparatively, state-of-the-art iris recognition methods [1][2][3] require that most of the iris codes should be matched.

How to define suitable global features to strengthen the robustness of local features based methods is not well addressed before, and it should be an important issue in future works. In addition, we think the global features should play a defining role in indexing of large scale iris databases.

\section{Acknowledgement}

This work is funded by research grants from the National Basic Research Program (Grant No. 2004CB318110), Natural Science Foundation of China (Grant No. 60335010, 60121302, 60275003, 60332010, 69825105) and the Chinese Academy of Sciences.

\section{References}

1. J. Daugman, "High Confidence Visual Recognition of Persons by a Test of Statistical Independence", IEEE Trans. Pattern Analysis and Machine Intelligence, Vol.15, No.11, pp.1148-1161, 1993.

2. L. Ma, T. Tan, Y. Wang, and D. Zhang, "Efficient Iris Recognition by Characterizing Key Local Variations", IEEE Trans. Image Processing, Vol. 13, No. 6, pp.739-750, 2004.

3. C. Sanchez-Avila, R. Sanchez-Reillo, "Two different approaches for iris recognition using Gabor filters and multiscale zero-crossing representation", Pattern Recognition, Vol. 38, No. 2, pp. 231-240, 2005.

4. Zhenan Sun, Yunhong Wang, Tieniu Tan, Jiali Cui, "Improving Iris Recognition Accuracy via Cascaded Classifiers", IEEE Transactions on Systems, Man, and Cybernetics-Part C: Applications and Reviews, Vol. 35, No. 3, pp.435-441, August 2005.

5. Topi Mäenpää, Matti Pietikäinen, "Texture analysis with local binary patterns”, Chapter 1, in C. Chen and P. Wang (eds) Handbook of Pattern Recognition and Computer Vision, 3rd ed, World Scientific, pp.197-216, 2005.

6. Michal Dobeš and Libor Machala, UPOL Iris Database, http://www.inf.upol.cz/iris/.

7. CASIA Iris Image Database, http://www.sinobiometrics.com.. 